\title{
Imaging of human brain tumor tissue by near-infrared laser coherence tomography
}

\author{
H. J. Böhringer • E. Lankenau • F. Stellmacher • \\ E. Reusche • G. Hüttmann • A. Giese
}

Received: 18 May 2008 / Accepted: 19 February 2009 / Published online: 3 April 2009

(C) Springer-Verlag 2009

\begin{abstract}
Introduction Intraoperative detection of residual tumor remains an important challenge in surgery to treat gliomas. New developments in optical techniques offer non-invasive high-resolution imaging that may integrate well into the workflow of neurosurgical operations. Using an intracranial glioma model, we have recently shown that time domain optical coherence tomography (OCT) allows discrimination of normal brain, diffusely invaded brain tissue, and solid tumor. OCT imaging allowed acquisition of $2 \mathrm{D}$ and $3 \mathrm{D}$ data arrays for multiplanar analysis of the tumor to brain interface. In this study we have analyzed biopsy specimens of human brain tumors and we present the first feasibility study of intraoperative OCT and post-image acquisition processing for non-invasive imaging of the brain and brain tumor.
\end{abstract}

This work contains parts of a doctoral thesis presented to the Faculty of Medicine by the first author Hans-Joachim Böhringer

\author{
H. J. Böhringer \\ Department of Surgery, St. Ansgar Clinics, \\ Bassum, Germany \\ E. Lankenau • G. Hüttmann \\ Institute for Biomedical Optics, University of Lübeck, \\ Lübeck, Germany \\ F. Stellmacher $\cdot$ E. Reusche \\ Department of Neuropathology, \\ University Hospital Schleswig-Holstein, \\ Lübeck, Germany \\ A. Giese $(\square)$ \\ Department of Neurosurgery, \\ Georg-August-University of Göttingen, \\ Robert-Koch-Strasse 40, \\ 37075 Göttingen, Germany \\ e-mail: alf.giese@med.uni-goettingen.de
}

Methods We used a Sirius 713 Tomograph with a superluminescence diode emitting light at a near infrared central wavelength of $1,310 \mathrm{~nm}$ and a coherence length of $15 \mu \mathrm{m}$. The light is passed through an optical mono mode fiber to a modified OCT adapter containing a lens system with a working distance of $10 \mathrm{~cm}$ and an integrated pilot laser. Navigation-registered tumor biopsies were imaged ex vivo and the intraoperative site of optical tissue analysis was registered by marker acquisition using a neuronavigation system.

Results Optical coherence tomography non-contact measurements of brain and brain tumor tissue produced B-scan images of $4 \mathrm{~mm}$ in width and 1.5-2.0 $\mathrm{mm}$ in depth at an axial and lateral optical resolution of $15 \mu \mathrm{m}$. OCT imaging demonstrated a different microstructure and characteristic signal attenuation profiles of tumor versus normal brain. Post-image acquisition processing and automated detection of the tissue to air interface was used to realign A-scans to compensate for image distortions caused by pulse- and respiration-induced movements of the target volume. Realigned images allowed monitoring of intensity changes within the scan line and facilitated selection of areas for the averaging of A-scans and the calculation of attenuation coefficients for specific regions of interest.

Conclusion This feasibility study has demonstrated that OCT analysis of the tissue microstructure and light attenuation characteristics discriminate normal brain, areas of tumor infiltrated brain, solid tumor, and necrosis. The working distance of the OCT adapter and the A-scan acquisition rate conceptually allows integration of the OCT applicator into the optical path of the operating microscopes. This would allow a continuous analysis of the resection plain, providing optical tomography, thereby adding a third dimension to the microscopic view and information on the light attenuation characteristics of the tissue. 
Keywords Glioma · Brain tumor - Optical coherence tomography $\cdot$ Intraoperative imaging

\section{Introduction}

Aggressive surgical resection results in prolonged survival of patients with a malignant glioma [22], may lower the risk of anaplastic progression in a low-grade glial tumor, and may thereby increase survival in these patients $[19,20]$. However, the intraoperative detection of residual tumor tissue, especially in a low-grade tumor, is difficult because of a low optical contrast between tumor and adjacent brain. Moreover, a glioma lacks a true tumor to brain interface, instead invading adjacent brain with single invasive cells migrating along preformed anatomical structures for several centimeters beyond the highly cellular margin of the tumor $[14,13,15]$. Intraoperative ultrasonography and intraoperative MRI provide excellent anatomical orientation and have demonstrated their value in the detection of gross residual tumor. However, the spatial resolution of ultrasound imaging in brain tissue is typically no higher than $150-250 \mu \mathrm{m}$. The spatial resolution of MRI is between 0.5 and $1 \mathrm{~mm}$, which is insufficient to resolve tissue microstructure or to discriminate between tissue alterations due to tumor infiltration and those as a result of surgery. Because of their complexity, neither technique can provide on-line imaging of the advancing resection cavity and the integration of these technologies into microsurgical instruments or operating microscopes is limited.

Optical coherence tomography (OCT) is an emerging biomedical imaging technology that has been applied to a wide range of biological, medical, and material investigations. Analogous to B-mode ultrasound, reflections of nearinfrared light are detected rather than sound, which offers non-invasive, real-time imaging in vivo. Imaging of solid tissues is possible up to $3 \mathrm{~mm}$ into the tissue depending on the light attenuation characteristics. Typically, spatial resolution of 5-15 $\mu \mathrm{m}$ can be obtained. Recent developments in retinal imaging have demonstrated a resolution of $3 \mu \mathrm{m}$; thus, it is now possible to differentiate individual retinal layers in vivo [11]. The first medical applications of OCT were in ophthalmology where imaging can be performed through the transparent media of the anterior eye and the retina $[16,17,25]$. OCT using optical sources at longer wavelengths have enabled imaging in highly lightscattering soft tissues, and its applications now include cardiology [18], gastroenterology [8, 28], urology [29, 40], dermatology [27, 33], dentistry [9], and the central nervous system $[2,3,6,7,12]$.

In ultrasonography the time delay of reflected sound waves is used to generate an image of tissue structures. Because of the extremely high velocity of light, a time delay of reflected light cannot be measured directly. Therefore, light is split into probe and reference light. The reference light and the probe light are combined by a beam splitter and registered at a detector. Interference of lowcoherence light only occurs when the optical path length of reference and probe are matched within the coherence length of the light source, which means that the difference between the optical path of the reference and the probe light determines the depth in the sample at which the magnitude of reflection is registered. Scanning the sample beam along a transverse axis can be performed to create a B-scan image and obtain two-, three-, and four-dimensional data arrays. This interferometer with fiber-optic couplers and beam scanning systems has been implemented to perform OCT measurements through microscopes, fiber-optic catheters and endoscopes [5, 30, 39], and hand-held probes [33].

We have recently demonstrated that OCT allows discrimination of normal brain, diffusely invaded brain tissue, and solid tumor in an experimental intracranial glioma mouse model [4]. These studies demonstrated that OCT imaging allowed 2D and 3D optical tomography of the tumor to brain interface, showing a detailed image of the tissue microstructures up to a tissue depth of 2-3 mm [4]. In this study we have investigated OCT imaging of human glioma biopsy specimens and present a first report of a clinical pilot evaluation of intraoperative time domain OCT imaging and post-acquisition image processing as a new intraoperative imaging modality.

\section{Materials and methods}

\section{Specimens and histology}

Specimens of human brain tumors were obtained at surgery under protocol \# 05-004 granted by the ethics committee of the University of Lübeck. Informed consent was given by patients $24 \mathrm{~h}$ prior to surgery. Tumor tissue was removed using standard microsurgical techniques and the resection site of individual tissue blocks was documented by marker acquisition using a vector vision2 neuronavigation system (BrainLab, Heimstetten, Germany), which allowed correlation of MRI signal characteristics and OCT B-scan images. For ex vivo OCT imaging the tissue was immediately placed on ice and the resection plain was imaged. Following analysis, the tissue was fixed in formalin $4.5 \%$ and was then paraffin embedded for standard histological processing. Histological sections were evaluated by the Department of Neuropathology.

Time domain optical coherence tomography

A Sirius 713 Tomograph (4Optics AG, Luebeck, Germany) developed at the Institute for Biomedical Optics, University 
of Lübeck, Germany was modified for intraoperative use. A superluminescence diode (SLD) served as a light source emitting light at a near infrared wavelength with a central wavelength of $1310 \mathrm{~nm}$ and a coherence length of $15 \mu \mathrm{m}$ (Fig. 1). The light is launched into an optical mono mode fiber, which guides the radiation to a modified OCT adapter containing a lens system with a working distance of $10 \mathrm{~cm}$ and an integrated pilot laser. The angle at which the laser enters the tissue should remain between $30^{\circ}$ and $150^{\circ}$ for optimal image information. Reflection artifacts from fluids on the tissue surface may occur at an exact angle of $90^{\circ}$ (Böhringer et al., manuscript in preparation). This allows measurements of brain tissue and brain tumor tissue in a no-touch technique and produces $2 \mathrm{D}$ B-scan-like images of $4 \mathrm{~mm}$ in width and $1.5-2.0 \mathrm{~mm}$ in depth, depending on the tissue characteristics. The image acquisition time at a resolution of 100 pixels $/ \mathrm{mm}$ was about $8 \mathrm{~s}$ for a $4-\mathrm{mm}$ scan line. The configuration used in this study provided axial and lateral optical resolutions of $15 \mu \mathrm{m}$.
Ex vivo imaging

Tissue specimens were obtained during resection of brain tumors and immediately placed on ice. Imaging was performed using a laboratory set-up of a Sirius 713 Tomograph with its OCT applicator mounted $2.5 \mathrm{~cm}$ above a computer-controlled $\mathrm{x} / \mathrm{y} / \mathrm{z}$ stage, which allowed accurate positioning of the tissue sample. The beam entering the tissue at a $90^{\circ}$ angle relative to the resection plain was focused and positioned to areas of interest using a pilot laser and 4-mm scan lines were imaged.

In vivo imaging

Intraoperative OCT analysis of surgical resection planes was performed in 9 patients (6 newly diagnosed glioblastomas WHO IV, 2 recurrent glioblastomas WHO IV, and 1 patient with an anaplastic astrocytoma WHO III), at different stages of resection of the tumor. Initially, an area

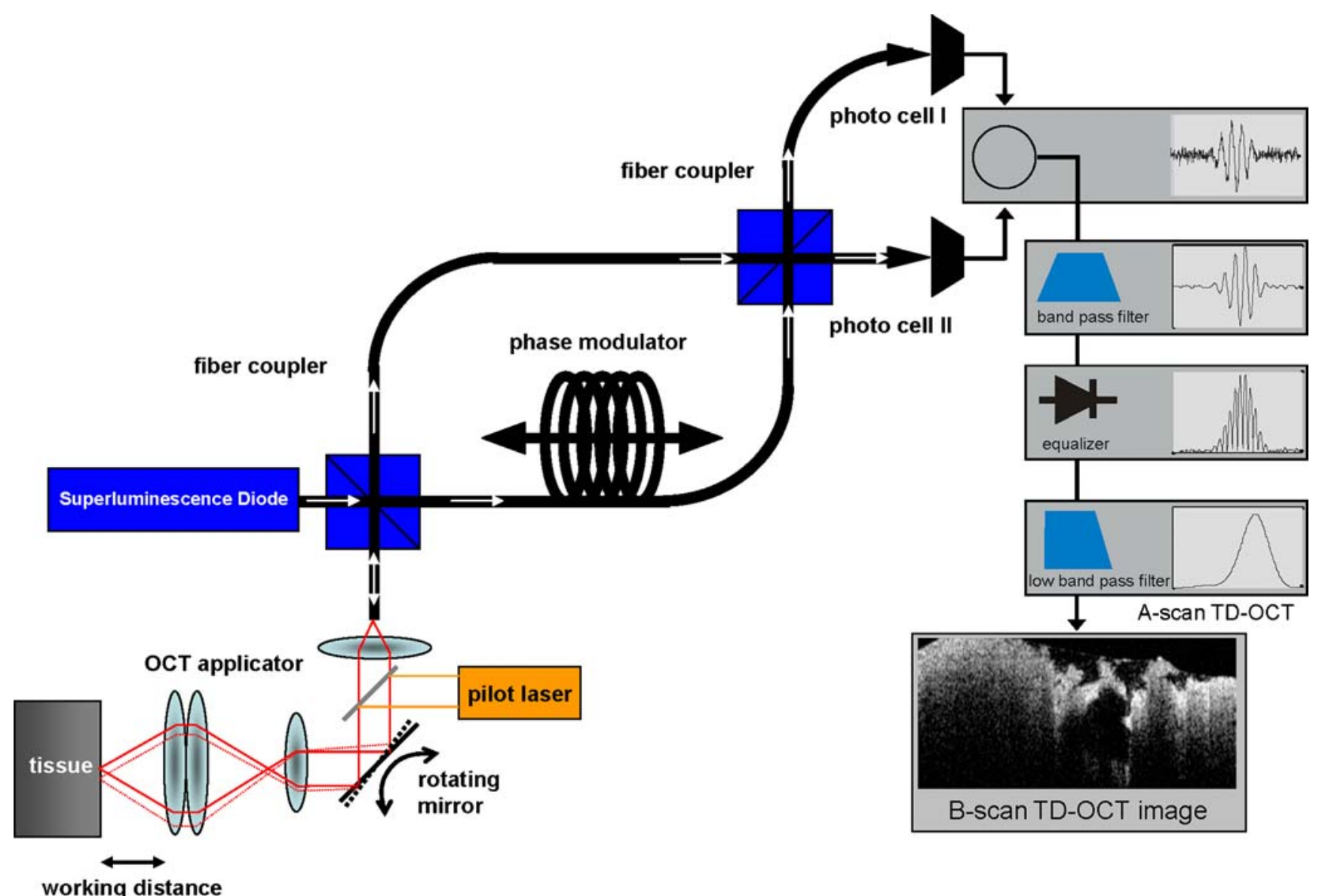

Fig. 1 Fiber optic interferometer implementation for time domain optical coherence tomography. A superluminescence diode (SLD) with a central wavelength of $1,300 \mathrm{~nm}$ is used as a light source. The light is passed through a beam splitter and sent to the sample and a phase modulator. Interference of low-coherence light only occurs when the optical path length of the phase modulator and the sample are matched. The sample beam can be scanned along a transverse axis to create a B-scan image and obtain two-, three-, and four-dimensional data arrays 
of solid tumor within the bulk tumor was selected using the neuronavigation and imaged with OCT. Then, during different stages of the resection, macroscopically distinct areas were selected for OCT imaging. Following a macroscopically complete resection arbitrarily selected areas of the wall of the resection cavity were imaged. For intraoperative imaging, the OCT applicator containing the lenses, the rotating mirror and a pilot laser, was mounted to a flexible arm attached to the operating table. For measurements of tumor tissue in situ or the walls of the resection cavity, the probe was placed above the craniotomy at a working distance of $10 \mathrm{~cm}$ and the target tissue volume was brought into the focal plain by adjusting the distance of the probe. A 4-mm scan line was measured at $0.5 \mathrm{~mm} / \mathrm{s}$. Because the imaging window of the tomography only covers a depth of $5 \mathrm{~mm}$, the geometry of the area selected for the 4-mm scan had to present a relatively level area. The steep portion of the resection cavity was therefore imaged using a horizontal orientation of the scan line. The scanned area was documented by marker acquisition using neuronavigation as above. A biopsy of the scanned tissue volume was taken and processed for histological evaluation as above. The stored OCT image files were reviewed by a blinded investigator and evaluated for light attenuation profile and intra-tissue microstructure.

\section{Results}

Ex vivo imaging of human brain tumor specimens by time domain optical coherence tomography

Tissue samples of normal cortex that were analyzed ex vivo showed a characteristic image with a fine line of an intense surface signal and a homogeneous decrease in the signal with increasing tissue depth (Fig. 2). In the cortex no prominent structures were identified within the tissue. An interpretable signal was obtained up to a tissue depth of $1.5 \mathrm{~mm}$. In contrast, specimens from glioblastomas showed a heterogeneous picture with a prominent microstructure of the tumor parenchyma clearly distinct from the signal of human cortex. Within the tumor parenchyma we found signal intense structures and areas of low signal intensity that interconnected to form larger structures. The latter were found predominantly in sites of tumor necrosis (see below). Imaging of a meningioma specimen and its dural insertion showed layering of the dura, thickening of the dura, and loss of layering closer to the tumor mass. Low signal intensity vascular elements were found at the tumor surface (Fig. 2). To correlate OCT imaging with the tumor morphology evident using a conventional operating microscope, biopsies of a glioblastoma were obtained at different stages of resection. The biopsy sites were documented by marker acquisition using a neuronavigation system to illustrate the anatomical region and the gross tumor structure at the site of analysis as well as the distance between the biopsy sites. Ex vivo OCT imaging of the specimens was performed immediately. Biopsies obtained from normal temporo-basal cortex using the view of the operating microscope anatomically most distant from the tumor mass showed signal characteristics similar to specimens of normal cortex, with a signal intense surface and a homogeneous decrease in the signal into the tissue depth. A prominent microstructure was not seen (Fig. 3a). A sample, which by neuronavigation appeared adjacent to, but not within the contrast-enhancing rim of the tumor, showed a loss of the intense surface signal and appearance of a
Fig. 2 Optical coherence tomography (OCT) imaging of native tissue obtained from surgical specimens of human cortex, a glioblastoma WHO grade $\mathrm{IV}$, and a meningioma, and its dural attachment site
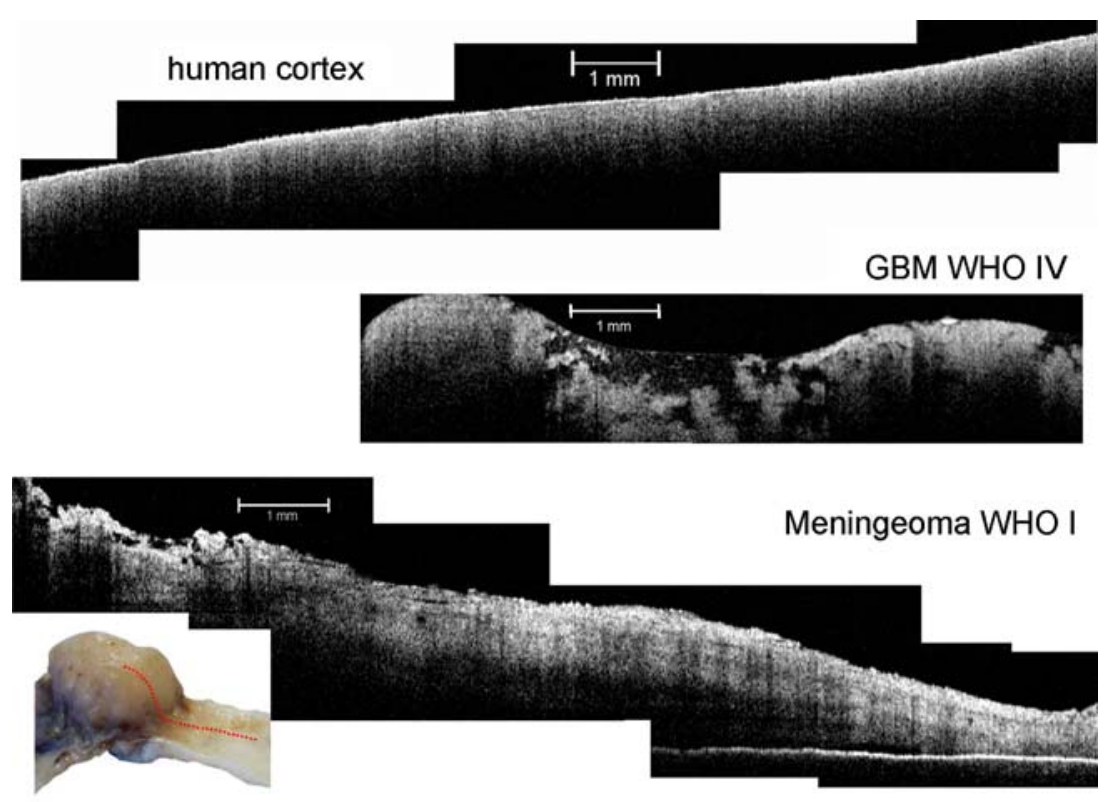


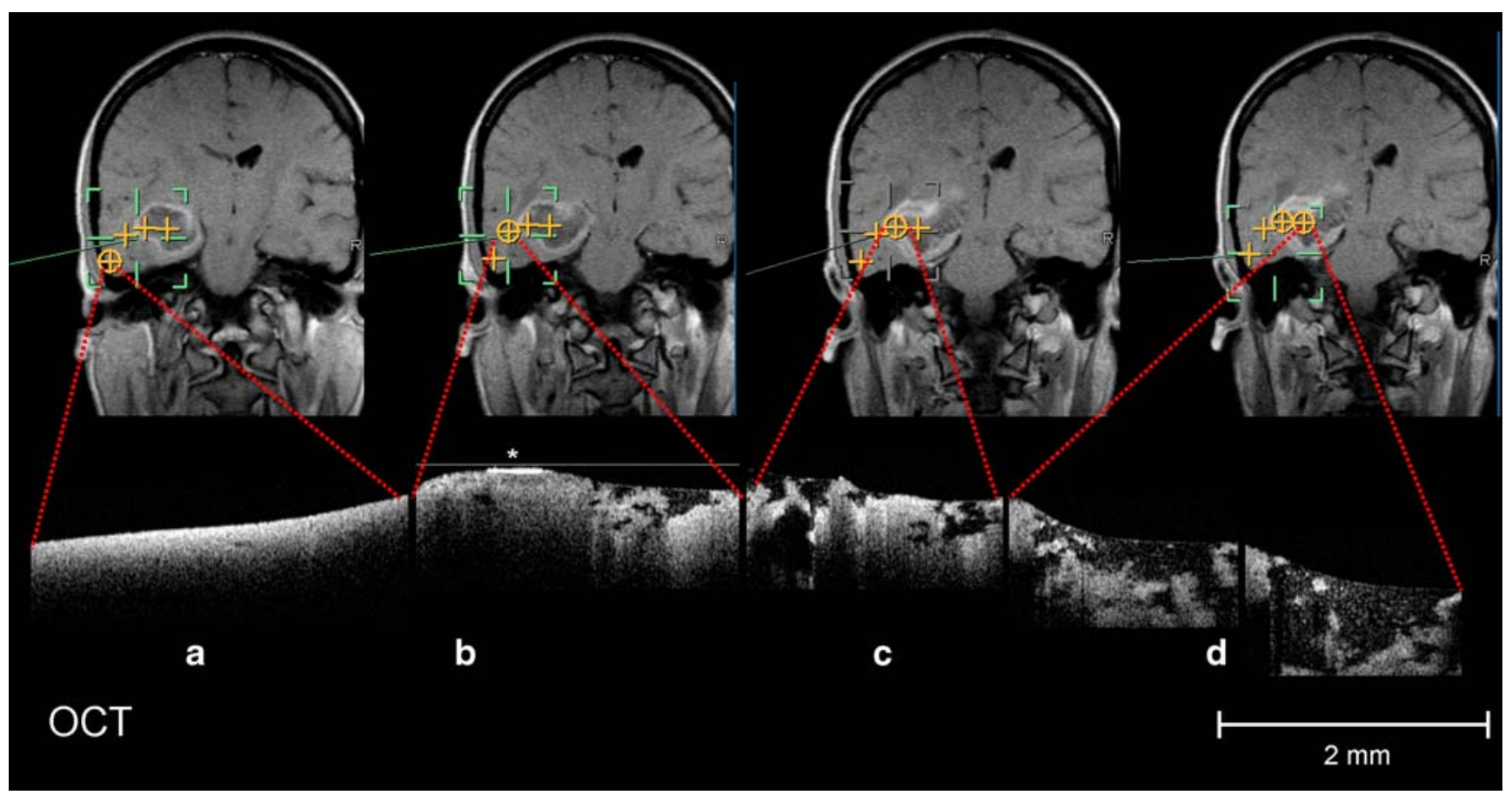

Fig. 3 During resection of a large right temporal glioblastoma WHO grade IV tissue samples were obtained and the biopsy site was documented by acquisition of marker points using a neuronavigation system. The tissue was immediately subjected to OCT analysis and the resection plain was imaged with no further processing of the tissue. a No-contact OCT imaging showed the different signal characteristics of the adjacent cortex, $\mathbf{b}$ a zone of signal abnormality close to the contrast-enhancing tumor presumably invaded brain, c the signal intense contrast-enhancing tumor mass, and $\mathbf{d}$ the inhomogeneous appearance of necrotic areas. Note, signal intense artefact due to fluid accumulation on the tissue surface (star) heterogeneous microstructure within the tissue (Fig. 3b). Tissue obtained from an area of abnormal tissue appearance by magnification of the operating microscope, which, according to intraoperative neuronavigation, corresponded to an area of contrast enhancement on preoperative MRI, showed a heterogeneous microstructure of high- and lowsignal intensity areas, focally resembling cavernous structures (Fig. 3c). Samples from the macroscopically easily identified central tumor necrosis contained more and larger low-intensity areas, some of which contained dot-like highintensity areas (Fig. 3d). B-scan OCT images consist of parallel A-scans, which may be averaged to calculate a light attenuation curve describing the signal intensity as a function of depth. This provides information on the light attenuation characteristics of the target volume. In a glioblastoma, ex vivo analysis of tissue obtained from an area of macroscopic tumor and adjacent brain showed that the averaging of distinct tumor areas resulted in individual light attenuation curves for tumor and adjacent brain (Fig. 4). An attenuation coefficient may be calculated from the log scale attenuation curves of areas with a homogeneous signal, so describing the linear part of the attenuation curve. The attenuation coefficient of macroscopic tumor $\left(32 \mathrm{~cm}^{-1}\right)$ was different from adjacent (presumably invaded) brain $\left(22 \mathrm{~cm}^{-1}\right)$. Areas of tumor necrosis with a

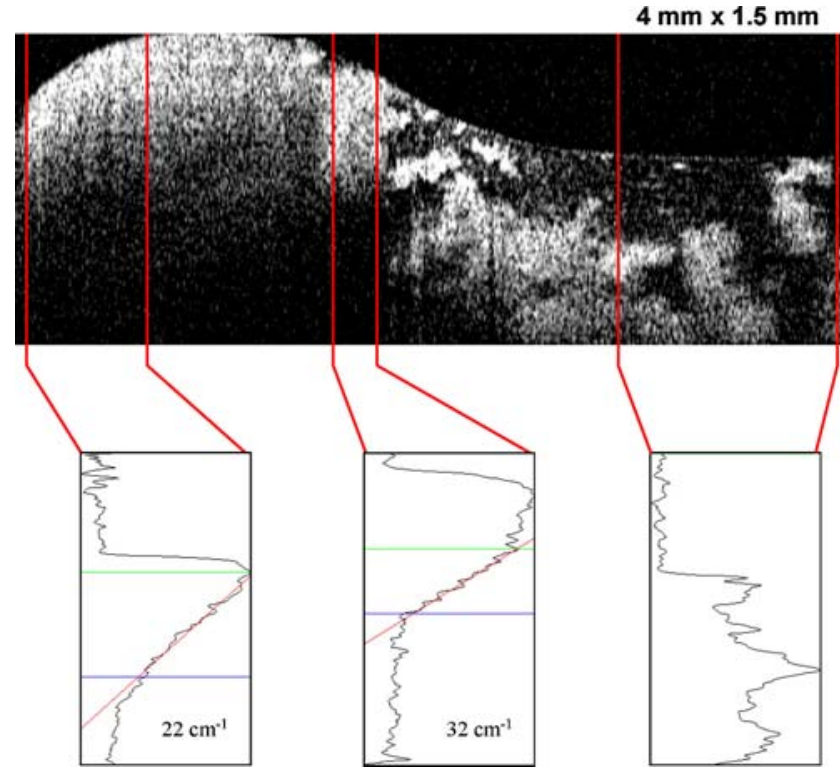

Fig. 4 Optical coherence tomography images consist of parallel Ascans along a scan line forming a two-dimensional B-scan image. Analysis of individual A-scans provides depth information on the signal intensity. By curve fitting of the signal intensity along the Ascan a scatter coefficient may be calculated. The shape of scatter curves in areas of brain adjacent to tumor, solid contrast-enhancing tumor, and tumor tissue with micronecrosis were significantly different 
heterogeneous microstructure of high- and low-intensity areas did not allow calculation of attenuation coefficients because a linear regression requires homogeneous signal distribution.

Intraoperative time domain optical coherence tomography

Optical tissue imaging of ex vivo specimens may be influenced by the loss of tissue perfusion and volume changes due to distortion of the three-dimensional tissue contexts. OCT imaging of the exposed brain during neurosurgical operations is challenged by the fact that the target volume follows a pulse and respiratory cycle. Intraoperative time domain OCT was performed using a flexible arm to position the optical probe above the craniotomy approximately $10 \mathrm{~cm}$ above the target volume. In a recurrent glioblastoma, a recraniotomy was performed and the brain surface, which macroscopically showed dural adhesions and scarring, was imaged. Four-millimeter scans at $0.5 \mathrm{~mm} / \mathrm{s}$ were obtained. The image of the target volume showed a characteristic distortion reflecting the pulse and respiratory cycle (Fig. 5). Automated detection of the surface structure of the tissue image was used to realign the A-scans. Realigned images allowed monitoring of intensity changes within the scan line and gave a clearer picture of the tissue microstructures, which also facilitated selection of areas for the averaging of A-scans and the calculation of attenuation coefficients.

To document the intraoperative OCT imaging site on preoperative MRI, the center of a scan line was visualized by a pilot laser and registered by marker acquisition using a neuronavigation system. OCT images were obtained from different regions of the operating field at different stages of resection (Fig. 6). Resection was completed after the analysis and the imaged tissue volume was fixed in formalin and processed for histological evaluation. In a recurrent glioblastoma, the histological evaluation demonstrated scarred cortical tissue, white matter invaded by tumor, highly cellular and highly vascularized solid tumor, and necrosis. The scarred cortical tissue on OCT imaging showed high signal intensity with a rapid loss of the signal in deeper tissue structures. Correspondingly, a high attenuation coefficient of $53 \mathrm{~cm}^{-1}$ was found (Fig. 6a). OCT analysis of cortex invaded by tumor showed a changed tissue microstructure and a decrease in the signal intensity. Because of the heterogeneous tissue structure, the attenuation coefficient was not analyzed by averaging the entire scan, but was measured in selected areas. The attenuation coefficient was significantly lower than in scarred cortex (20-27 $\mathrm{cm}^{-1}$ ) (Fig. 6b). When the resection proceeded to the solid and highly vascularized tumor, OCT analysis showed a heterogeneous microstructure of the tumor parenchyma and a light attenuation coefficient ranging
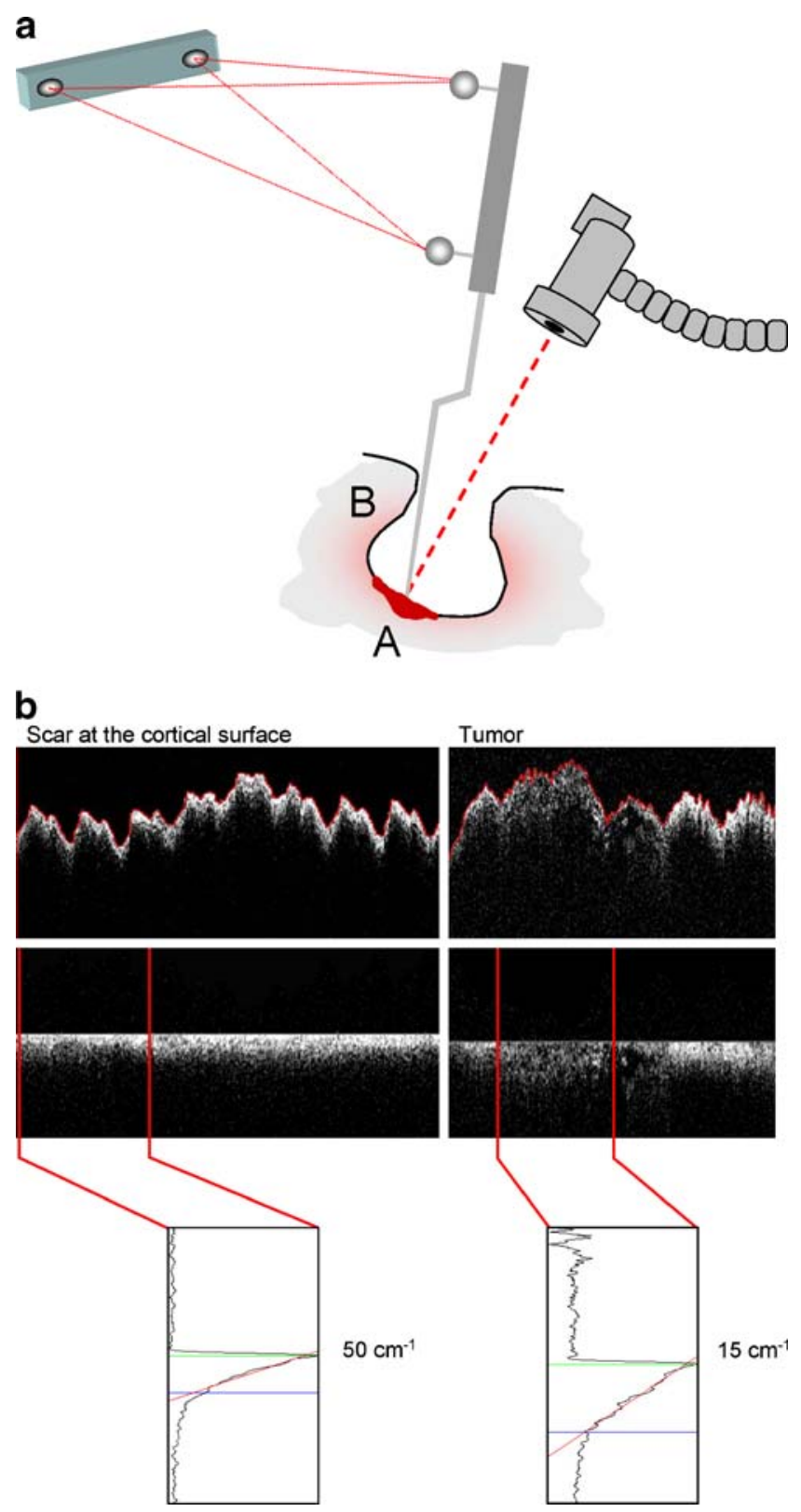

Fig. 5 a Illustration of intraoperative OCT measurements. The OCT applicator containing a lens system, a rotating mirror, and a pilot laser, was positioned over the resection cavity using a flexible arm attached to the operating table. Measurements were performed at a fixed working distance of $10 \mathrm{~cm}$ by adjusting the distance of the probes to the target tissue. A 4-mm scan line was measured at $0.5 \mathrm{~mm} / \mathrm{s}$. The analysis was started in an area of highly cellular known tumor $(A)$ and continued to analysis of the walls of the resection cavity $(B)$. The scanned area was documented by point registration of the center of the scan line using a neuronavigation system. A biopsy of the scanned tissue volume was taken and processed for histological evaluation. b Intraoperative OCT imaging during resection of a recurrent glioblastoma (WHO grade IV). The exposed brain follows a volume change induced by the respiratory and arterial cycle, which at scan times of $1 \mathrm{~mm}$ per second for $1.5 \mathrm{~s}$ results in characteristic distortion of the tissue. Automated detection of the tissue surface (red line) allows realignment of the A-scans, which can be used to reduce the tissue distortion 

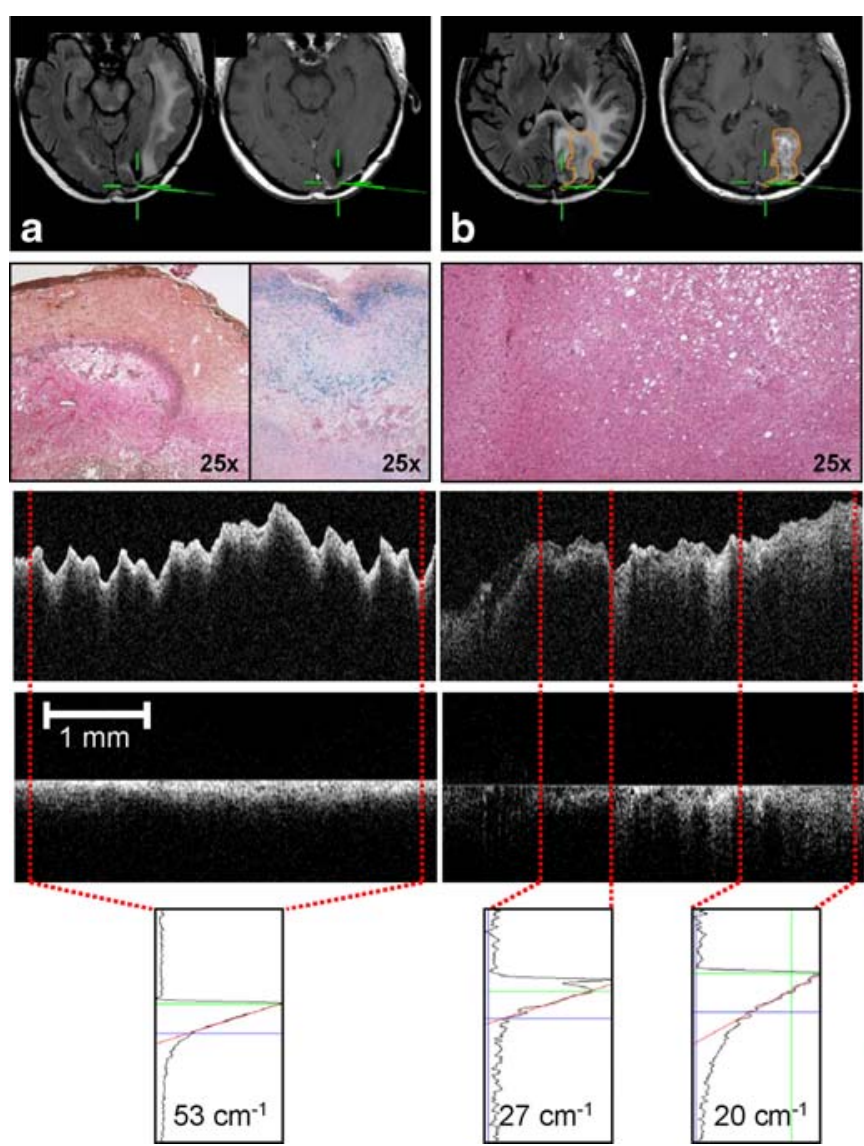

Fig. 6 Intraoperative OCT imaging during resection of glioblastoma (WHO grade IV), which recurred after initial surgery and radiation. A pilot laser was used to focus the OCT probe on the target volume. The center of the scan line was registered by the acquisition of marker points using a neuronavigation system. a Collagen-rich cortical scar $\mathrm{H} \& \mathrm{E}$ stained (left) with deposition of iron pigmented macrophages

from 9 to $20 \mathrm{~cm}^{-1}$. Histology showed a highly cellular tumor and neovascularization in this area (Fig. 6c). OCT analysis of tumor necrosis showed similar findings to those of our ex vivo evaluation, with low-intensity areas and focal cavernous-like structures. The attenuation coefficient in these areas was low (Fig. 6d).

\section{Clinical pilot study}

A clinical pilot study of intraoperative OCT during the resection of malignant gliomas was carried out in 9 patients (6 newly diagnosed glioblastomas, WHO IV; 2 recurrent glioblastomas, WHO IV, and 1 case of an anaplastic astrocytoma, WHO III). At different stages of the microsurgical tumor resections, 42 sites (selected as described in Materials and methods) were OCT imaged and biopsies were obtained for routine histological evaluation. The stored OCT image files were scored as "normal light attenuation," "loss of normal light attenuation," and "loss of normal light attenuation with a pathological tissue micro-
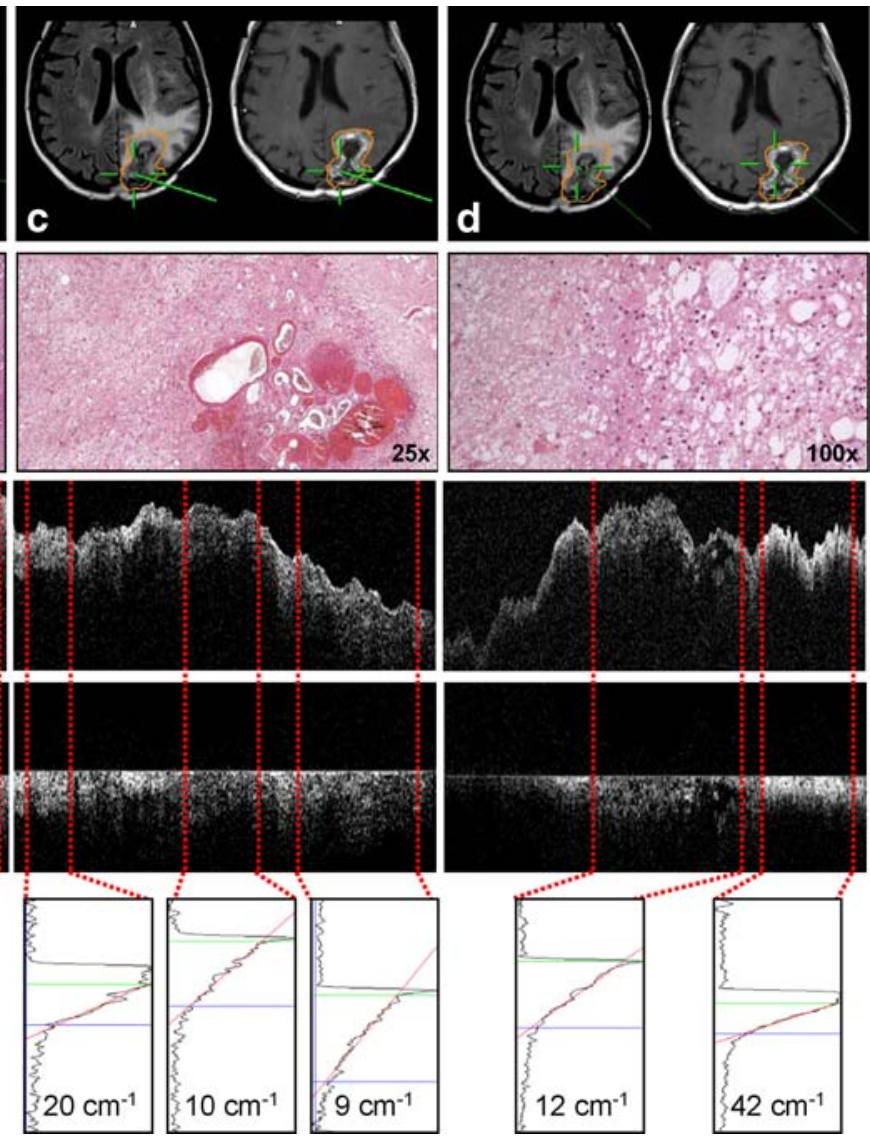

(right; Berlin blue reaction). b Micro-cystic changes within the tumor infiltration zone. c Highly cellular tumor with vascular proliferations and pleomorphic tumor cells. d Pleomorphic tumor cells (right) and tumor necrosis (left) within the center of the tumor (b-d; H\&E staining)

structure" by a blinded investigator not present in the operating room. The biopsy samples were H\&E stained and scored "no tumor," "diffuse tumor infiltration," and "solid tumor" by a neuropathologist blinded to the OCT findings. According to histological evaluation, 15 samples represented solid tumor tissue. In all of these, the light attenuation profile characteristic of normal brain was lost and a pronounced microstructure, which is typically absent in normal brain, was identified. In 15 samples of histologically diffuse tumor infiltration, OCT showed an abnormal light attenuation profile in all of the intraoperative images. In 9 of these analyses OCT also demonstrated an abnormal tissue microstructure similar to the findings in solid tumor. Twelve biopsies contained no tumor by histology. In 9 of these samples OCT showed homogeneous tissue with the typical light attenuation profile of normal brain. However, in 3 samples of histologically normal brain OCT suggested a loss of the normal light attenuation pattern (Fig. 7). In these samples histology demonstrated areas of microhemorrhage. There was a clear correlation between the 


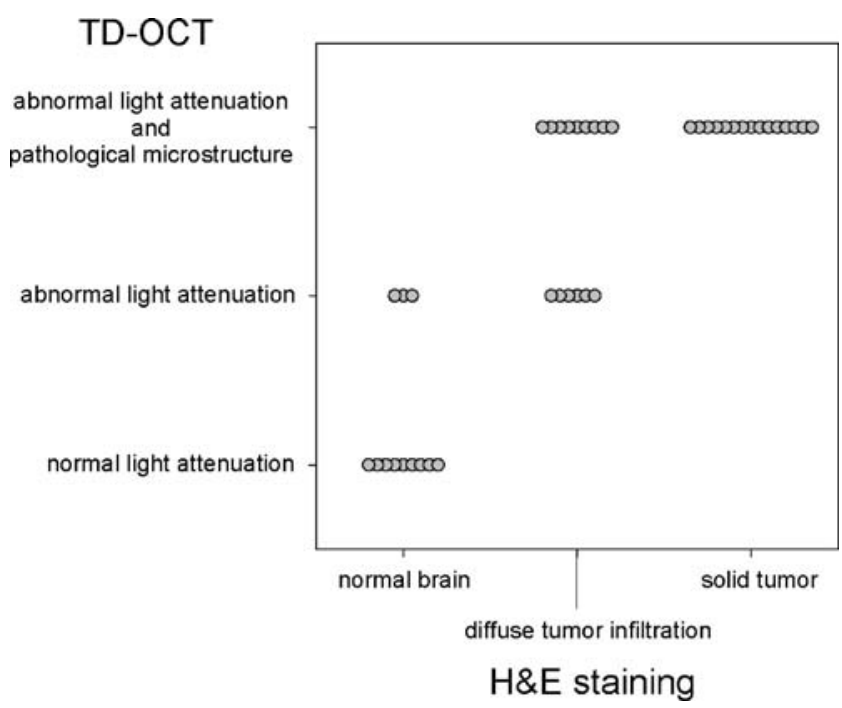

Fig. 7 Correlation of intraoperative OCT analysis and postoperative standard histology. Forty-one optical tissue analyses were performed during the operations of 9 malignant gliomas. A blinded investigator scored the stored OCT image files and the scores were compared with those of the blinded evaluation of H\&E-stained biopsy specimens

scoring of the optical tissue analysis and the result of the histology ( $\chi^{2}$ test; $\left.r=0.99\right)$.

\section{Discussion}

The resection of intrinsic brain tumors is challenged by the fact that these tumors do not have a defined edge delineating the tumor mass from the adjacent normal brain. Since the early days of the surgical treatment of gliomas, neurosurgeons have used the guidance of anatomical landmarks, the color and texture of tumor tissue, and the tendency to hemorrhage from resection plains. Although recognition of solid tumor or tumor necrosis based on these criteria is relatively safe, even with the aid of an operating microscope it has remained difficult to delineate highly cellular tumor or densely invaded brain from normal or lightly invaded brain. The highly cellular part of the tumor on MRI roughly corresponds to the area of gadolinium contrast enhancement [21]. The extent of removal of this contrast-enhancing tumor correlates with survival of the patient [22]. However, the surgeon's estimate of "complete removal of visible tumor" may only correlate with "clearance of enhancement on MRI" in about $30 \%$ of cases, which reflects the difficulty of the intraoperative detection of residual tumor [1]. Therefore, techniques for the intraoperative detection of highly cellular areas at the edge of the resection cavity may improve the chances of the complete removal of a tumor and may influence overall survival of a patient treated with a malignant glioma.
Intraoperative ultrasound has become a routine aid to the resection of brain tumors. After removal of the bone flap, transdural or transcortical ultrasonography delineates the solid tumor mass from normal brain [37]. However, the spatial resolution of 4- to $10-\mathrm{MHz}$ probes applicable to neurosonography is only approximately $200 \mu \mathrm{m}$, which is well below that needed to detect the tissue microstructure or cellular elements. Furthermore, areas of tumor infiltration and the variable zone of edema surrounding brain tumors are poorly differentiated by ultrasound. Another wellknown phenomenon in intraoperative neurosonography is that surgical trauma to normal brain tissue results in timedependent changes of the tissue morphology and its sonographic signal characteristics. Micro-hemorrhage, changes in tissue perfusion, and edema may appear similar on ultrasound to tumor tissue, which limits the value of neurosonography in the detection of residual tumor [37]. Intraoperative MRI provides excellent anatomical orientation at a spatial resolution of $0.5-1.0 \mathrm{~mm}$. During the resection of a brain tumor, intraoperative MRI can visualize residual tumor and can be used to update the data for intraoperative navigation systems [35]. Nevertheless, tissue changes as a consequence of microtrauma to normal brain tissue seem to result in phenomena similar to those observed in ultrasound and non-specific enhancement of gadolinium contrast at the edge of a resection cavity was described early in the evaluation of this technology [34].

Conceptually, these problems may be solved by early analysis of the resection edge before contusion results in non-specific imaging findings. This, however, would require a technique that allows continuous analysis of the advancing edge of the resection cavity. Because of their complexity, ultrasound and intraoperative MRI require a pause in the course of the resection and can only be used for intermittent analyses, and realistically for only a limited number of investigations during the operation. In contrast, optical tissue analysis offers some advantages for integration into neurosurgical equipment and procedures. Because optical coherence tomography can be performed as a noninvasive and no-contact technique over focal distances of several centimeters it can be integrated into neurosurgical operating microscopes [23]. Potentially, this would allow a continuous analysis of the tissue in view by a tomographic image of the resection edge as it is created.

Few studies have been undertaken to apply OCT to imaging of the central nervous system anatomy or disease [7, 26, 31]. Recently, ultrahigh resolution optical coherence tomography resolved the brain tissue morphology from a single cell level to a whole animal brain ex vivo and in vivo [2]. The same authors then showed that ex vivo highresolution optical coherence tomography of formalin-fixed specimens of human cortex, meningioma, and ganglioglioma has the potential to discriminate between normal 
brain and brain tumor biopsies [3]. We have shown that OCT in highly light-scattering central nervous system tissue provides tomographic images of up to $2 \mathrm{~mm}$ in depth [4]. In an experimental glioma model in mouse brain, OCT detected highly cellular tumor of less than $1 \mathrm{~mm}^{3}$ and delineated the signals from areas of brain invaded by tumor from histologically normal brain at high resolution [4]. In experimental gliomas, OCT discriminated the growth pattern of invasive and non-invasive tumors in mouse brain and allowed the multiplanar reconstruction of the tumor/ brain interface by three-dimensional reconstruction of OCT image stacks [7]. Ex vivo OCT analysis of biopsies taken from the wall of a resection cavity following a microsurgically complete resection of an astrocytoma (WHO III) was able to detect microscopic residual tumor (Böhringer et al., submitted). This suggests that further developments in intraoperative OCT may provide a tool for controlling the extent of resection. In this study we demonstrated the first in vivo OCT imaging of human brain and brain tumor tissue.

The interpretation of the tomographic images relied on the microstructure of the target tissue and on the light attenuation characteristics. Sections through normal brain showed a characteristic attenuation profile. In this study, time domain optical coherence tomography demonstrated that human cortex and white matter showed a similar light attenuation profile and a homogeneous parenchymal signal was found in normal brain. In brain adjacent to tumor, the attenuation profile of the brain parenchyma was altered and within highly cellular tumor the attenuation profile was entirely changed. The attenuation characteristic of tissue may be analyzed by averaging A-scans, which allows calculation of an attenuation curve [32, 33]. In homogeneous tissues such as normal brain or highly cellular tumor, regression analysis of the linear part of the attenuation curve allows calculation of a light attenuation coefficient, which was different in brain parenchyma and highly cellular tumor. However, calculation of a light attenuation coefficient was not possible for images of tumor containing areas of necrosis. The heterogeneous distribution of high and low intensity along the axis of an A-scan resulted in attenuation curves with no linear loss of the signal. Further studies of the cellular correlates of attenuation characteristics in brain and brain tumors will be needed to show if attenuation curve analysis can provide a basis for detailed tissue analysis.

Optical coherence tomography-based no-contact imaging of brain tissue during neurosurgical procedures is challenged by the fact that after the opening of the dura the target volume follows the respiratory and arterial cycle, resulting in movements of several millimeters in amplitude. Our feasibility study of intraoperative OCT has shown that the movement of the exposed brain results in a typical distortion of the image. While the target volume remained within the imaging window of the Sirius 713 Tomograph, the interpretation of B-scan images was more difficult than for ex vivo tissue analysis. This limitation was overcome by post-acquisition image processing using software developed by the Institute for Biomedical Optics at the University of Lübeck. Automated identification of the airto-tissue intensity change at the tissue surface allowed realignment of the A-scans composing the B-scan image. Alternatively, decreased scan times may eliminate motion artifacts. In an experimental glioma model we recently demonstrated the spectral domain implication of OCT [4]. Spectral domain OCT retrieves the depth information by cross-spectral density reconstruction of the interferogram through detection of the interference signal as a function of wavelength. This allows image acquisition rates of three images per second [10, 36, 38]. Recently, an ultra-highspeed implementation of this technique in an experimental application in ophthalmology showed elimination of motion artifacts and the need to re-align A-lines, thus enabling rapid and accurate $3 \mathrm{D}$ mapping of the retina and the optic nerve [24].

This study has demonstrated that no-contact OCT provides non-invasive imaging information that can differentiate between areas of tumor and adjacent brain in malignant brain tumors in vivo. Our clinical pilot study represents the first application of this technology to tumor resection in neurosurgery. The correlation between histologically solid tumor or densely invaded brain with the loss of a normal light attenuation profile and the occurrence of a pathological tissue microstructure suggests that OCT is highly sensitive to tumor and the loss of the normal histoarchitecture. However, our data also indicate that OCT characteristics may be influenced by surgical artifacts. An abnormal light attenuation profile was observed in three samples of histologically normal brain. These samples histologically contained micro-hemorrhage, most likely reflecting tissue changes due to surgical trauma. We have previously observed in animal studies that the light attenuation profile at a resection edge within normal brain changes in a time-dependent manner, indicating that both exposure and tissue contusion alter the OCT signal (unpublished data). Further, we have demonstrated that micro- and macro-hemorrhage at the resection edge, surgical coagulation, the use of hemostyptics and other surgical materials influence the appearance on OCT. Some of these effects we have identified as irreversible (Böhringer et al., manuscript in preparation). This again highlights the necessity for early analysis of the resection margin before such changes. The experimental set-up used in this feasibility study required significant time for positioning of the OCT probe and measurements. This precluded a full scan of the resection edges and limited this 
study to the correlation of OCT imaging profiles and corresponding histology for selected sites at different stages of the resection. We have recently integrated OCT into the optical path of an operating microscope [23]. This prototype will now allow a continuous analysis of the resection margin and provide information on the light attenuation characteristics of the tissue. Such a set-up would integrate optical tissue analysis into the workflow of neurosurgical operations without the need to discontinue the resection or with requirements for additional instruments.

Acknowledgement This study was supported by grants of the University Hospital of Schleswig-Holstein (AG), The Kreitz Foundation (AG) and the Future Investment Program of Schleswig-Holstein (AG, GH, EL).

\section{References}

1. Albert FK, Forsting M, Sartor K, Adams HP, Kunze S (1994) Early postoperative magnetic resonance imaging after resection of malignant glioma: objective evaluation of residual tumor and its influence on regrowth and prognosis. Neurosurgery 34:45-61. doi:10.1097/00006123-199401000-00008

2. Bizheva K, Unterhuber A, Hermann B, Povazay B, Sattmann H, Drexler W et al (2004) Imaging ex vivo and in vitro brain morphology in animal models with ultrahigh resolution optical coherence tomography. J Biomed Opt 9:719-724. doi:10.1117/ 1.1756920

3. Bizheva K, Unterhuber A, Hermann B, Povazay B, Sattmann H, Fercher AF et al (2005) Imaging ex vivo healthy and pathological human brain tissue with ultra-high-resolution optical coherence tomography. J Biomed Opt 10:011006. doi:10.1117/1.1851513

4. Böhringer HJ, Boller D, Leppert J, Knopp U, Lankenau E, Reusche E et al (2006) Time domain and spectral domain optical coherence tomography in the analysis of brain tumor tissue. Lasers Surg Med 38:588-597. doi:10.1002/1sm.20353

5. Böhringer HJ, Lankenau E, Rohde V, Hüttmann G, Giese A (2006) Optical coherence tomography for experimental neuroendoscopy. Minim Invasive Neurosurg 49:269-275. doi:10.1055/ s-2006-954574

6. Boppart SA (2003) Optical coherence tomography: technology and applications for neuroimaging. Psychophysiology 40:529541. doi:10.1111/1469-8986.00055

7. Boppart SA, Brezinski ME, Pitris C, Fujimoto JG (1998) Optical coherence tomography for neurosurgical imaging of human intracortical melanoma. Neurosurgery 43:834-841. doi:10.1097/ 00006123-199810000-00068

8. Bouma BE, Tearney GJ, Compton CC, Nishioka NS (2000) Highresolution imaging of the human esophagus and stomach in vivo using optical coherence tomography. Gastrointest Endosc 51:467474. doi:10.1016/S0016-5107(00)70449-4

9. Colston BW, Everett MJ, Da Silva LB, Otis LL, Stroeve P, Nathal $\mathrm{H}$ (1998) Imaging of hard- and soft-tissue structure in the oral cavity by optical coherence tomography. Appl Opt 37:3582-3585. doi:10.1364/AO.37.003582

10. De Boer JF, Cense B, Park BH, Pierce MC, Tearney GJ, Bouma BE (2003) Improved signal-to-noise ratio in spectral-domain compared with time-domain optical coherence tomography. Opt Lett 28:2067-2069. doi:10.1364/OL.28.002067

11. Drexler W (2001) Ultrahigh-resolution optical coherence tomography. Invest Ophthalmol Vis Sci 42:1993-2003
12. Fujimoto JG, Pitris C, Boppart SA, Brezinski ME (2000) Optical coherence tomography: an emerging technology for biomedical imaging and optical biopsy. Neoplasia 2:9-25. doi:10.1038/sj. neo.7900071

13. Giese A, Westphal M (1996) Glioma invasion in the central nervous system. Neurosurgery 39:1-18. doi:10.1097/00006123199608000-00001

14. Giese A, Rief MD, Loo MA, Berens ME (1994) Determinants of human astrocytoma migration. Cancer Res 54:3897-3904

15. Giese A, Bjerkvig R, Berens ME, Westphal M (2003) The cost of migration. Invasion of malignant gliomas and implications for treatment. J Clin Oncol 21:1624-1636. doi:10.1200/ JCO.2003.05.063

16. Hee MR, Puliafito CA, Wong C, Duker JS, Reichel E, Rutledge B et al (1995) Quantitative assessment of macular edema with optical coherence tomography. Arch Ophthalmol 113:325-332

17. Hee MR, Izatt JA, Swanson EA, Huang D, Schuman JS, Lin CP et al (1995) Optical coherence tomography of the human retina. Ophthalmology 102:217-229

18. Jang IK, Bouma BE, Kang DH, Park SJ, Park SW, Seung KB et al (2002) Visualization of coronary atherosclerotic plaques in patients using optical coherence tomography: comparison with intravascular ultrasound. J Am Coll Cardiol 39:604-609. doi:10.1016/S0735-1097(01)01799-5

19. Keles GE, Anderson B, Berger MS (1999) The effect of extent of resection on time to tumor progression and survival in patients with glioblastoma multiforme of the cerebral hemisphere. Surg Neurol 52:371-379. doi:10.1016/S0090-3019(99)00103-2

20. Keles GE, Lamborn KR, Berger MS (2001) Low-grade hemispheric gliomas in adults: a critical review of extent of resection as a factor influencing outcome. J Neurosurg 95:735-745

21. Kelly PJ, Daumas-Duport C, Kispert DB, Kall BA, Scheithauer BW, Illig JJ (1987) Imaging-based stereotaxic serial biopsies in untreated intracranial glial neoplasms. J Neurosurg 66:865-874

22. Lacroix M, Abi-Said D, Fourney DR, Gokaslan ZL, Shi W, DeMonte F et al (2001) A multivariate analysis of 416 patients with glioblastoma multiforme: prognosis, extent of resection, and survival. J Neurosurg 95:190-198

23. Lankenau E, Klinger D, Winter C, Malik A, Müller HH, Oelckers S, Pau H-W, Just T, Hüttmann G (2007) Combining Optical Coherence Tomography (OCT) with an operating microscope. In: Buzug TM, Holz D, Weber S, Bongartz J, Kohl-Bareis M, Hartmann U (eds) Advances in medical engineering. Springer, Berlin, pp 343-348

24. Nassif N, Cense B, Park BH, Pierce MC, Yun SH, Bouma $\mathrm{BE}$ et al (2004) In vivo high-resolution video-rate spectraldomain optical coherence tomography of the human retina and optic nerve. Opt Express 12:367-376. doi:10.1364/ OPEX.12.000367

25. Puliafito CA, Hee MR, Lin CP, Reichel E, Schuman JS, Duker JS et al (2004) Imaging of macular diseases with optical coherence tomography. J Biomed Opt 9:47-74. doi:10.1117/1.1629679

26. Roper SN, Moores MD, Gelikonov GV, Feldchtein FI, Beach NM, King MA et al (1998) In vivo detection of experimentally induced cortical dysgenesis in the adult rat neocortex using optical coherence tomography. J Neurosci Methods 80:91-98. doi:10.1016/S0165-0270(97)00202-1

27. Schmitt JM, Yadlowsky MJ, Bonner RF (1995) Subsurface imaging of living skin with optical coherence microscopy. Dermatology 191:93-98

28. Tearney GJ, Brezinski ME, Southern JF, Bouma BE, Boppart SA, Fujimoto JG (1997) Optical biopsy in human gastrointestinal tissue using optical coherence tomography. Am J Gastroenterol 92:1800-1804

29. Tearney GJ, Brezinski ME, Southern JF, Bouma BE, Boppart SA, Fujimoto JG (1997) Optical biopsy in human urologic tissue using 
optical coherence tomography. J Urol 157:1915-1919. doi:10.1016/S0022-5347(01)64900-0

30. Tearney GJ, Brezinski ME, Bouma BE, Boppart SA, Pitris C, Southern JF et al (1997) In vivo endoscopic optical biopsy with optical coherence tomography. Science 276:2037-2039. doi:10.1126/science.276.5321.2037

31. Uma Maheswari R, Takaoka H, Homma R, Kadono H, Tanifuji M (2002) Implementation of optical coherence tomography (OCT) in visualization of functional structures of cat visual cortex. Opt Commun 202:47-54. doi:10.1016/S0030-4018(02)01079-9

32. Welzel J, Bruhns M, Wolff HH (2003) Optical coherence tomography in contact dermatitis and psoriasis. Arch Dermatol Res 295:50-55. doi:10.1007/s00403-003-0390-y

33. Welzel J, Reinhardt C, Lankenau E, Winter C, Wolff HH (2004) Changes in function and morphology of normal human skin: evaluation using optical coherence tomography. Br J Dermatol 150:220-225. doi:10.1111/j.1365-2133.2004.05810.x

34. Wirtz CR, Knauth M, Staubert A, Bonsanto MM, Sartor K, Kunze S et al (2000) Clinical evaluation and follow-up results for intraoperative magnetic resonance imaging in neurosurgery. Neurosurgery 46:1112-1122. doi:10.1097/00006123-200005000-00017

35. Wirtz CR, Knauth M, Stamov M, Bonsanto MM, Metzner R, Kunze S et al (2002) Clinical impact of intraoperative magnetic resonance imaging on central nervous system neoplasia. Tech Neurosurg 7:326-331. doi:10.1097/00127927-200207040-00012

36. Wojtkowski M, Leitgeb R, Kowalczyk A, Bajraszewski T, Fercher AF (2002) In vivo human retinal imaging by Fourier domain optical coherence tomography. J Biomed Opt 7:457-463. doi: $10.1117 / 1.1482379$

37. Woydt M, Krone A, Becker G, Schmidt K, Roggendorf W, Roosen K (1996) Correlation of intra-operative ultrasound with histopathologic findings after tumor resection in supratentorial gliomas. A method to improve gross total tumor resection. Acta Neurochir (Wien) 138:1391-1398. doi:10.1007/BF01411117

38. Wojtkowski M, Bajraszewski T, Gorczynska I, Targowski P, Kowalczyk A, Wasilewski W et al (2004) Ophthalmic imaging by spectral optical coherence tomography. Am J Ophthalmol 138:412-419. doi:10.1016/j.ajo.2004.04.049
39. Xie T, Xie H, Fedder GK, Pan Y (2003) Endoscopic optical coherence tomography with a modified microelectromechanical systems mirror for detection of bladder cancers. Appl Opt 42:6422-6426. doi:10.1364/AO.42.006422

40. Zagaynova EV, Streltsova OS, Gladkova ND, Snopova LB, Gelikonov GV, Feldchtein FI et al (2002) In vivo optical coherence tomography feasibility for bladder disease. J Urol 167:1492-1496. doi:10.1016/S0022-5347(05)65351-7

\section{Comments}

Achieving a gross total resection in glioma surgery is still a distinct challenge in modern neurosurgery. Intraoperative imaging mainly applying ultrasound and magnetic resonance tomography was implemented in the recent years to achieve this goal more reliably. While intraoperative imaging acts as immediate intraoperative quality control additional multi-modal navigation prevents too extensive resections, preserving neurological function.

The authors have demonstrated an alternative method that might become a convenient possibility to directly monitor the ongoing course of a resection. Optical coherence tomography (OCT) seems to be suitable to analyze the immediate resection border and be able to discriminate between healthy and pathological tissue. The authors are to be encouraged to intensify their work especially also in respect to the intraoperative application, to correlate intraoperative findings to postoperative resection extent, so that in the long-term it can be decided whether OCT allows to achieve enlarged and more completed resections. Of course a lot of challenges will have to be met, until OCT will be able to allow a 3-D online analysis of the extent of a resection covering the whole tumor extent. The presented results confirm that OCT might become a promising technological addition to the neurosurgical armamentarium.

Christopher Nimsky

Marburg, Germany 